\title{
Cabezal compacto para un control inteligente de la bionutrición y el riego
}

\section{Martínez Tornero, José Francisco}

1 jtornero@nutricontrol.com

\section{Resumen:}

En los últimos años, la demanda de los consumidores y mercados hacia productos más sanos y cultivados de manera respetuosa con el medio ambiente, ha hecho que la agricultura experimente una evolución hacia sistemas más eficientes, precisos, tecnificados y sostenibles, para lo que es necesario poder disponer del control y de los datos para optimizar el uso de todos los recursos empleados en la producción.

Estos cambios han generado nuevas demandas para aquellas empresas que somos lideres en le desarollo de todo tipo de soluciones para la tecnificación del campo y la mayoría de estos retos gracias al uso de la tecnología, son resueltos de manera exitosa, mejorando los procesos exixtentes.

Otros aspectos que hemos identificado consecuencia, en muchos casos, de esa tecnificación son: (i) la necesidad de un mayor conocimento para el manejo de todos los datos que se generán, (ii) la aparición de diferentes sistemas de recogida de datos, (iii) el uso de nuevos fertilizantes orgánicos y bio estimulantes más respetuosos con el medio ambiente pero a la vez más dificles de manejar en los sistemas de riego, (iv) sistemas de cultivo intensivos y con variedades más exigentes, (v) las nuevas exigencias de los nuevos consumidores y la legislación vigente, mucho más exigentes con los recursos empleados en la producción, (vi) unido al precio de la energía y el agua que se ha incrementado de forma considerable (además del deficit de esta última), y (vii) todo ello sin dejar de perder rentabilidad en los cultivos y a la vez, ser respetuosos con el medio natural.

Por todo lo mencionado, se nos planteo el reto de aportar una solución integral que pueda mejorar o resolver en gran medida, las nuevas exigencias y permitir evolucionar hacia una agricultura mucho más sostenible y rentable. De ahí, y con la experiencia internacional de más de 30 años en el sector, decidimos desarrollar un sistema compacto e inteligente que intregrara todos los dispositivos necesarios para el manejo de forma autónoma y precisa del riego y la fertirrigación. Facilmente exportable y sencillo de montar, incorporandole una gran cantidad de sensórica que nos permitiera obtener una gran cantidad de datos que nos ayudaran a tomar las decisiónes más oportunas en cada monento para marcar una estrategia de manejo más rentable y adaptada a los nuevos productos y, sobre todo, nos marcamos el reto de integrar diferentes dispositivos bajo una única plataforma, con el fin de mejorar la experiencia del usuario final de forma intuitiva y amigable, eliminando así la problemática de tener que gestionar plataformas diferentes para dispositivos que tienen el mismo fin.

Tambien incorporamos la opción de poder compartir los datos generados por el sistema inteligente con otras plataformas o con los propios sistemas de gestión de las explotaciones agrícolas ya que estamos convencidos de que la comunicación abierta nos hace avanzar a todos. 
Palabras clave: sensórica; manejo del riego; digitalización de la agricultura; agricultura de precisión 


\section{Cabezal compacto para un control inteligente de la bionutrición y el riego.}

\section{Problemática abordada}

Nos encontramos la realidad de una nueva agricultura, mucho más digital, con un mayor requerimiento de información, de datos y, sobre todo, el reto de conseguir mantener los mismos rendimientos, pero bajo el paraguas de la sostenibilidad.

Las explotaciones agrícolas ya se comportan como una fábrica en la que debemos conocer todo aquello que se está utilizando (fertilizantes, agua, mano de obra, etc) todo aquello que requiere la planta y sobre todo poder medir y controlar aquello que se aporta para conseguir una producción sostenible.

Este modelo de agricultura sostenible, también es un reto para los equipos que tradicionalmente controlaban y aportaban todo lo necesario para los cultivos, por diversos motivos:

- Las dotaciones de agua son cada vez menores.

- La calidad del agua es menos óptima para los cultivos.

- El coste del agua se ha incrementado en los últimos años como consecuencia de su déficit y por la incorporación de agua desalada.

- Los suelos son más pobres en M.O.

- Las limitaciones en los aportes de fertilizantes convencionales, principalmente nitrogenados..

- El uso de nuevos abonos orgánicos y bioestimulantes mejoradores del suelo, con densidades y texturas diferentes a los convencionales, con volúmenes de aporte diferentes y por lo general, de un mayor coste.

- El perfil de agricultor está cambiando y empieza a valorar poder disponer de más tiempo para su vida personal y exige un mayor control y precisión a tiempo real.

- La aparición de diferentes sistemas ocasionando que las explotaciones dispongan de sistemas distintos que no interactúan entre ellos y no permiten disponer de un control integral del cultivo.

- El aumento de cultivos de alto valor y también más sensibles y exigentes en necesidades hídricas y requerimientos nutricionales (berries, aguacate, etc) junto con sistemas de cultivo más intensivos.

- La legislación también está evolucionando y empieza a ser más restrictiva y exigente en las aportaciones de agua, fertilizantes y materias activas para el control de plagas y enfermedades.

- El consumidor final también ha variado y empieza a exigir y valorar como se ha producido y que recursos se han consumido en aquello que van a comprar (huella hídrica). 
Todo lo anterior, ha requerido aportar soluciones mucho más precisas y completas que permitan a los agricultores abordar este nuevo escenario, pudiendo manejar todos los elementos de una instalación de riego de una forma integrada y precisa para disponer en todo momento de un control total del riego, la fertirrigación y el estado fitosanitario del cultivo.

\section{Descripción de la innovación}

Ante este nuevo reto, Nutricontrol decidió abordar la problemática para estar nuevamente del lado de una agricultura de precisión y sostenible, poniendo en valor y aglutinando todo el conocimiento, tecnología y experiencia acumulada en los últimos 30 años en el control de riego, el clima y la fertirrigación.

La prioridad fue buscar una solución holística a todo lo expuesto en el apartado anterior y englobarlo todo bajo una única plataforma y equipo compacto de fácil instalación y manejo.

Para ello, desarrollamos todo un sistema inteligente y compacto alimentado con diferentes tipos de tecnología para un control total de todo aquello que influye de manera directa sobre la rentabilidad de un cultivo y el manejo de la explotación (Figura 1).

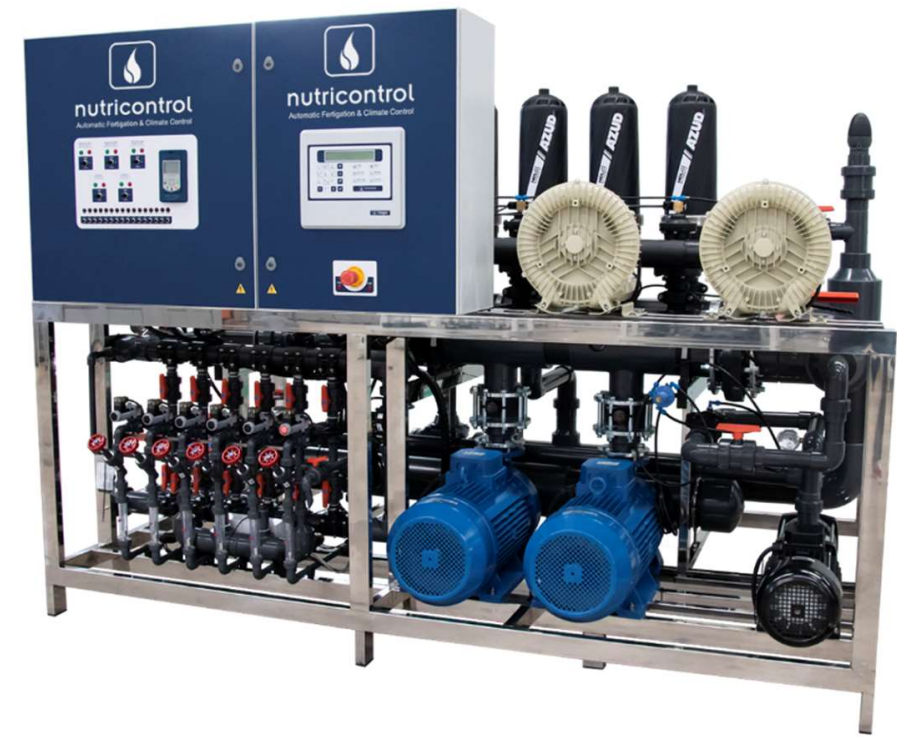

Figura 1. Solución de cabezal compacto inteligente.

El equipo desarrollado integra, bajo una única estructura compacta, todos los elementos necesarios para un control inteligente de todo el sistema de riego, fertirrigación y clima:

- Controlador de riego (Mithra Riego; Figura 2) capaza de manejar hasta 3 cabezales de riego de forma totalmente independientes, comandar hasta 200 válvulas ya sea por cable o de forma inalámbrica y controlar todos los elementos de la instalación. También es posible ampliar las prestaciones del mismo, únicamente a través de un código sin necesidad de cambiar el equipo o mandarlo a fábrica. 


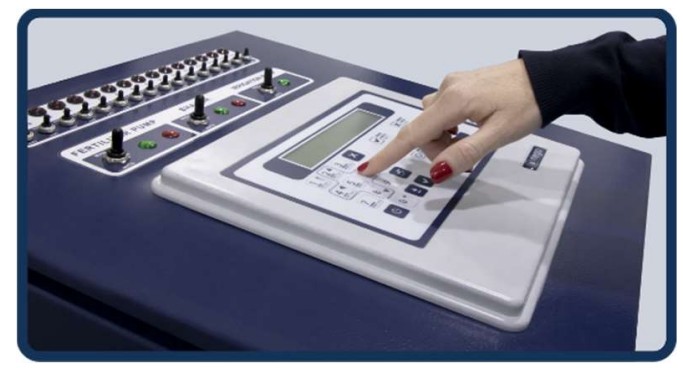

Figura 2. Controlador de riego Mithra

- Sistema de bombeo para presurizar la instalación siempre manejado con variadores que controla el mismo sistema de control de riego (Mithra Riego) con diferentes consignas de presión dependiendo del sector de riego.

- Sistema de filtrado de anillas aire - agua (Azud) dimensionado según las características de la instalación, fuente de agua y los volúmenes de agua a tratar.

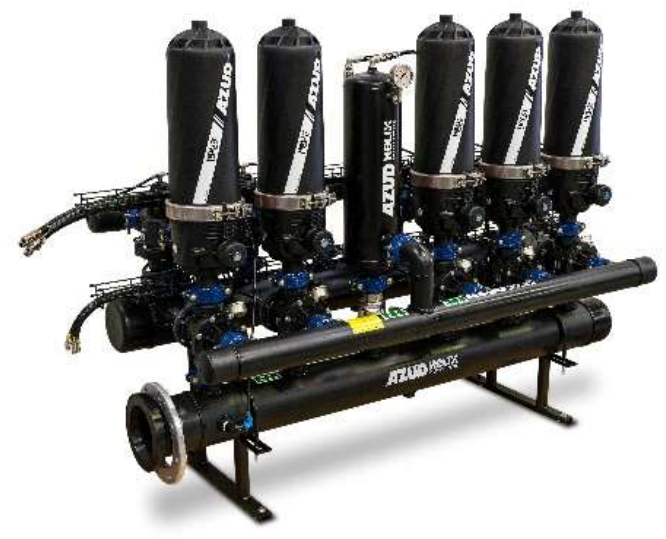

Figura 3. Sistema de filtrado de anillas aire - agua

- Contador de agua con emisor de pulsos para controlar los volúmenes de riego aportados y permitir un riego por volumen. Podemos disponer de los volúmenes de agua consumidos por cada sector o válvula.

- Equipo de fertirrigación con hasta 9 líneas de inyección que controla de forma automática el pH y la CE de hasta 20 recetas de abonado diferentes. Sistema multi inyección para poder aportar diferentes abonos a la vez y controlando en todo momento el $\mathrm{pH}$ de la mezcla para evitar precipitados.

- Sistema de inyección analógico: la inyección de los fertilizantes podemos controlarla con dos sistemas dependiendo del caudal a inyectar.

Si es necesario inyectar cantidades inferiores a $600 \mathrm{~L} / \mathrm{h}$, se controlará de forma automática con una nueva válvula motorizada (NUCON) desarrollada bajo las especificaciones de Nutricontrol para un control mucho más preciso y lineal de la $\mathrm{CE}$, el $\mathrm{pH}$ y el aporte de los fertilizantes. 
Si los caudales de inyección son mayores, podemos llegar a una inyección de hasta 1800 1/h por cada línea de inyección.

En ambos casos, hemos desarrollado un sistema de inyección que permite inyectar una mayor cantidad de abono y que, además, está diseñado para poder trabajar con abonos orgánicos, eliminando los riegos de obturaciones del sistema como consecuencia de las densidades, impurezas y texturas de estos abonos orgánicos y bioestimulantes.

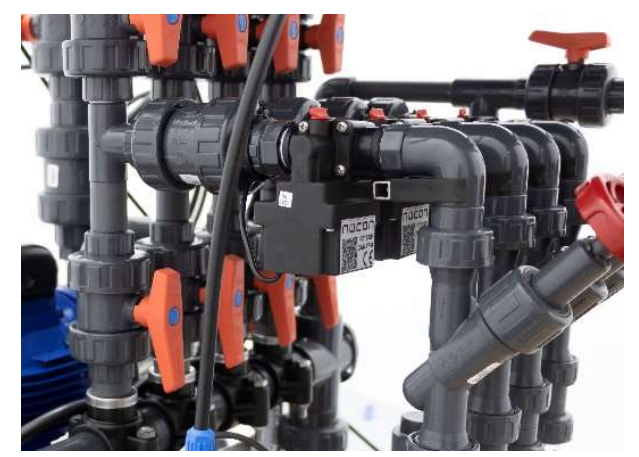

Figura 4. Sistema de inyección con válvulas Nucon.

- Contadores de abono por ultrasonidos. Estos contadores nos permiten realizar un abonado mucho más preciso, conociendo y aplicando exactamente las dosis necesarias en cada momento. Combinado junto con el contador de abono, podemos realizar un abonado por volumen y lo más importante, repartirlo durante todo el tiempo de riego para un reparto más uniforme a lo largo del bulbo húmedo.

- Sensores de suelo: El equipo permite programar el riego en función a parámetros de suelo obtenidos a través de diferentes sensores que midan la humedad del suelo (\%) o el potencial matricial. También podemos tener lectura de la $\mathrm{T}^{\mathrm{a}} \mathrm{y}$ la $\mathrm{CE}$ del suelo y cálculos como el agua disponible, punto de recarga, punto de marchitez, etc.

- Sensores de clima: Podemos también programar el riego en función a factores ambientales como la radiación o como la ETo o la ETc. Y cálculos como el DPV, punto de rocío, horas frio, DH, etc

- Con los valores de los sensores de clima, también disponemos de sistemas predictivos para ciertas enfermedades y ciertos cultivos, integral térmica, etc.

- Imágenes de satélite, que permiten disponer de valores como el NDVI, índice usado para estimar la cantidad, calidad y desarrollo de la vegetación con base a la medición de la intensidad de la radiación de ciertas bandas del espectro electromagnético que la vegetación emite o refleja y nos permite identificar problemas o nos ayuda a tomar decisiones de por dónde empezar a comprobar.

También podemos disponer de índices de humedad, integral térmica o necesidades de riego. 


\section{Congreso Nacional de Riegos CARTAGENA 2021}

- Comunicación web: Toda la información generada, que registrada en un servidor en la nube pudiendo consultar y cambiar parámetros desde cualquier dispositivo móvil om Pc con acceso a internet.

- Comunicación con otros dispositivos: Otra de las ventajas de disponer de todos los datos en la nube, es que podemos comunicarlos con otros dispositivos instalados en la explotación e interactuar entre ellos. Es posible que el agricultor disponga de sistemas de monitoreo (datalogger) y que la información recogida por estos dispositivos, pueda ser compartida bajo la misma plataforma que gestiona el riego, un mismo usuario y sin necesidad de tener que acceder a diferentes plataformas con usuarios y formas de operar diferentes.

- Compartir los datos con otros sistemas: El sistema desarrollado por Nutricontrol permite compartir los datos generados a través de la API (Application Programming Interface) para poder incorporarlo al sistema SCADA de cada cliente y poder hacer un uso más eficiente de los datos.

\section{Resultados obtenidos}

Con la integración de todos los sistemas y tecnologías anteriormente descritas, hemos conseguido dar solución a muchos de los problemas que aparecían a la hora de poder implementar tecnología que nos ayude a una agricultura de mayor precisión y sostenibilidad.

Entre los problemas que se han podido solventar o mejorar se encuentran:

\section{- 3.1. Una mayor internacionalización de las soluciones tecnológicas;}

El hecho de haber estandarizado la fabricación y haberlo hecho escalable y compacto, permite poder exportar la tecnología de una manera más sencilla y fácil para el cliente final. Dispone de un único proveedor y de un producto que el cliente lo recibe montado, probado y programado de fábrica, lo que reduce los imprevistos del montaje y la mano de obra en un 35\% con respecto a una instalación tradicional. Ya que en ocasiones es difícil encontrar mano de obra especializada. Además de asegurarnos la compatibilidad de funcionamiento de todos los elementos.

\section{- 3.2. Una mayor eficiencia energética;}

El uso de variadores para controlar las bombas de riego de forma autónoma desde el equipo y con consignas diferentes de presión dependiendo del sector, combinado con el uso de sensores de humedad de suelo o potencial matricial, optimiza el consumo eléctrico y el diseño de la instalación de riego, ya que permite modificar las estrategias de riego en función a dichos sensores, optimizar los tiempos y frecuencias de riego, consiguiendo ahorros energéticos de entre un 20 y un $40 \%$ con respecto al manejo de un cabezal de riego tradicional.

El optimizar los tiempos de riego, ajustarlos y no aplicar el riego más de lo estrictamente necesario, conlleva también un aumento de la vida útil de la instalación y de todos sus elementos, ya que estamos reduciendo las horas de funcionamiento de cada uno de ellos. 


\section{Congreso Nacional de Riegos CARTAGENA 2021

Tabla 1. Ejemplo de ahorro energético obtenido en 1 ha de cítricos

\begin{tabular}{cccc}
\hline Cultivo & $\begin{array}{c}\text { Coste energé- } \\
\text { tico } € / \text { ha }\end{array}$ & $\begin{array}{c}\text { \% ahorro } \\
\text { energético }\end{array}$ & $\begin{array}{c}\text { Ahorro } \\
(€ / \text { ha })\end{array}$ \\
\hline $\begin{array}{c}\text { Cítricos } \\
1 \text { ha }\end{array}$ & 917,28 & $35 \%$ & 321,0 \\
\hline
\end{tabular}

Precio del kW/h: 0,15€

- 3.3. Optimización del agua de riego;

La instalación de sensores de suelo para medir el agua útil disponible, la capacidad de campo, punto de marchitez, unto de recarga y conocer la profundidad del perfil de riego, permite conocer la disponibilidad y el consumo necesario para el cultivo en cada momento y en función a su estado fenológico. El uso de estos sensores de suelo, junto con el de variables climáticas que nos permiten conocer y calcular parámetros como la Etc, junto con el uso de contadores, permiten modificar los tiempos, frecuencias y dosis de agua de riego, pudiendo aportar lo estrictamente necesario en cada momento del ciclo del cultivo, evitando perdidas por escorrentías o percolación.

También nos permite conocer cuando está activo el sistema radicular y conocer el momento óptimo de aplicación de los fertilizantes.

El ahorro de agua con el uso de sensores de suelo y clima, permite ahorrar en general, entre un 20 y un $40 \%$ con respecto a los planes de riego tradicionales. 
Tabla 2. Ahorro hídrico con sensorización en diferentes cultivos

\begin{tabular}{llc}
\multicolumn{1}{c}{ CULTIVO } & \multicolumn{2}{c}{$\begin{array}{c}\text { NECESIDADES HÍDRI- } \\
\text { CAS } \\
\text { Ahorro 20\% (€/ha) }\end{array}$} \\
\hline AGUACATE & 3.400 & 680 \\
\hline ALMENDRO SETO & 2.600 & 520 \\
\hline ARÁNDANOS & 2.280 & 456 \\
\hline CIRUELA & 2.000 & 400 \\
\hline FRESA & 2.280 & 456 \\
\hline LIMÓN & 2.400 & 480 \\
\hline MANZANA & 2.960 & 592 \\
\hline MELOCOTÓN & 2.800 & 560 \\
\hline MELÓN & 2.400 & 480 \\
\hline NARANJO & 2.800 & 560 \\
\hline OLIVO & 1.600 & 320 \\
\hline PERA & 1.600 & 320 \\
\hline UVA & 2.480 & 496 \\
\hline PATATA & 1.200 & 240 \\
\hline
\end{tabular}

*Precio del agua $0,4\left(€ / \mathrm{m}^{3}\right)$

También el uso de sistemas de filtrado de alta eficiencia (aire/agua) permite disminuir los tiempos de limpieza, consiguiendo un ahorro de hasta un $80 \%$ del agua empleada en la limpieza con respecto a sistemas de filtrado estándar.

\subsection{Ahorro de fertilizantes}

El poder combinar en las lecturas diferentes valores de CE en la solución nutritiva de abonado, los contadores de abono en cada línea de inyección y los valores de $\mathrm{CE}$ en suelo a través de sensores de suelo, permite ajustar el aporte de fertilizantes según las necesidades de cada momento del cultivo. Controlar la CE de una solución nutritiva de abonado es fundamental para mantener niveles elevados de rentabilidad y productividad, ya que determinados niveles de CE dificultan la absorción de los abonos disminuyendo la productividad, incluso pudiendo llegar a ocasionar contaminación de las aguas subterráneas o un empobrecimiento del suelo. 
Tabla 3. Ahorro obtenido por el control de la CE para diferentes cultivos

\begin{tabular}{|c|c|c|c|}
\hline \multirow{3}{*}{ CULTIVO } & \multicolumn{3}{|c|}{$\mathrm{CE}$} \\
\hline & \multirow{2}{*}{$\begin{array}{c}\mathrm{CE} \\
\text { ideal }\end{array}$} & \multicolumn{2}{|c|}{ Pérdidas $(25 \%)$} \\
\hline & & $\mathrm{CE}$ & $€ /$ ha \\
\hline AGUACATE & 0,9 & 1,7 & 5.276 \\
\hline $\begin{array}{l}\text { ALMENDRO } \\
\text { SETO } \\
\end{array}$ & 1,0 & 1,9 & 2.164 \\
\hline $\begin{array}{l}\text { ARÁNDA- } \\
\text { NOS }\end{array}$ & 0,7 & 1,4 & 14.736 \\
\hline CIRUELA & 1,0 & 1,9 & 4.098 \\
\hline FRESA & 0,7 & 1,2 & 15.218 \\
\hline LIMÓN & 1,1 & 2,2 & 3.927 \\
\hline MANZANA & 1,0 & 2,2 & 6.290 \\
\hline $\begin{array}{l}\text { MELOCO- } \\
\text { TÓN } \\
\end{array}$ & 1,1 & 1,9 & 4.759 \\
\hline MELÓN & 1,5 & 3,8 & 2.979 \\
\hline NARANJO & 1,1 & 2,2 & 1.843 \\
\hline OLIVO & 1,8 & 3,7 & 939 \\
\hline PERA & 1,0 & 2,2 & 3.695 \\
\hline UVA & 1,0 & 2,7 & 6.914 \\
\hline PATATA & 1,1 & 2,5 & 3.652 \\
\hline
\end{tabular}

*Precios percibidos por los agricultores 2019/2020 (MAPA)

$\mathrm{Al}$ igual ocurre con el $\mathrm{pH}$, es fundamental mantener niveles ácidos en el abonado (5,5-6,5) para conseguir que la mayor parte de los elementos nutritivos estén disponibles para ser absorbidos por la planta.

\subsection{Eliminación de las obsturaciones del sistema y mayor presición de inyección;}

Uno de los retos que nos encontrábamos cuando se requería el aporte de abonos orgánicos, eran los problemas de obturación del sistema de fertirrigación sobre todo a nivel de valvulería y emisores.

Para evitar obturaciones del sistema de inyección y aplicación de los fertilizantes, se desarrolló una válvula (Figura 5) de un paso de líquido mayor a las utilizadas hasta el momento, pero sin perder precisión en la inyección y control de la $\mathrm{CE}$ y el $\mathrm{pH}$. 


\section{Congreso Nacional de Riegos CARTAGENA 2021

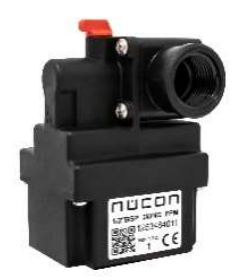

Figura 5. Válvula de control de inyección (Nucon)

La válvula dispone de un control analógico y lineal, mucho más preciso y estable que el control por pulsos utilizado comúnmente. Incluso con un sistema de seguridad de cierre en el caso de un corte de corriente eléctrico que evitaría que la válvula se quedara abierta ocasionado el vaciado de los tanques de abonado.

Esta válvula permite un control muy preciso de caudales de abono de entre 50 y $600 \mathrm{~L} / \mathrm{h}$ por cada línea de inyección, ideal para culticos sensibles a los cambios de $\mathrm{CE} \mathrm{o} \mathrm{pH.}$

Con este sistema de inyección, combinado con el sistema de filtrado aire/agua, resolvimos el problema de las obturaciones del sistema de inyección con el uso de abonos orgánicos y aumentamos la precisión en el control continuo de la CE.

\subsection{Reducción de los tratamientos y la aplicación de materias activas;}

El poder medir parámetros como la Ta , la HR (\%), radiación, la humectación en hoja, etc, nos permite desarrollar modelos predictivos de enfermedades lo que nos puede llevar a realizar los tratamientos únicamente en los momentos de mayor probabilidad del desarrollo.

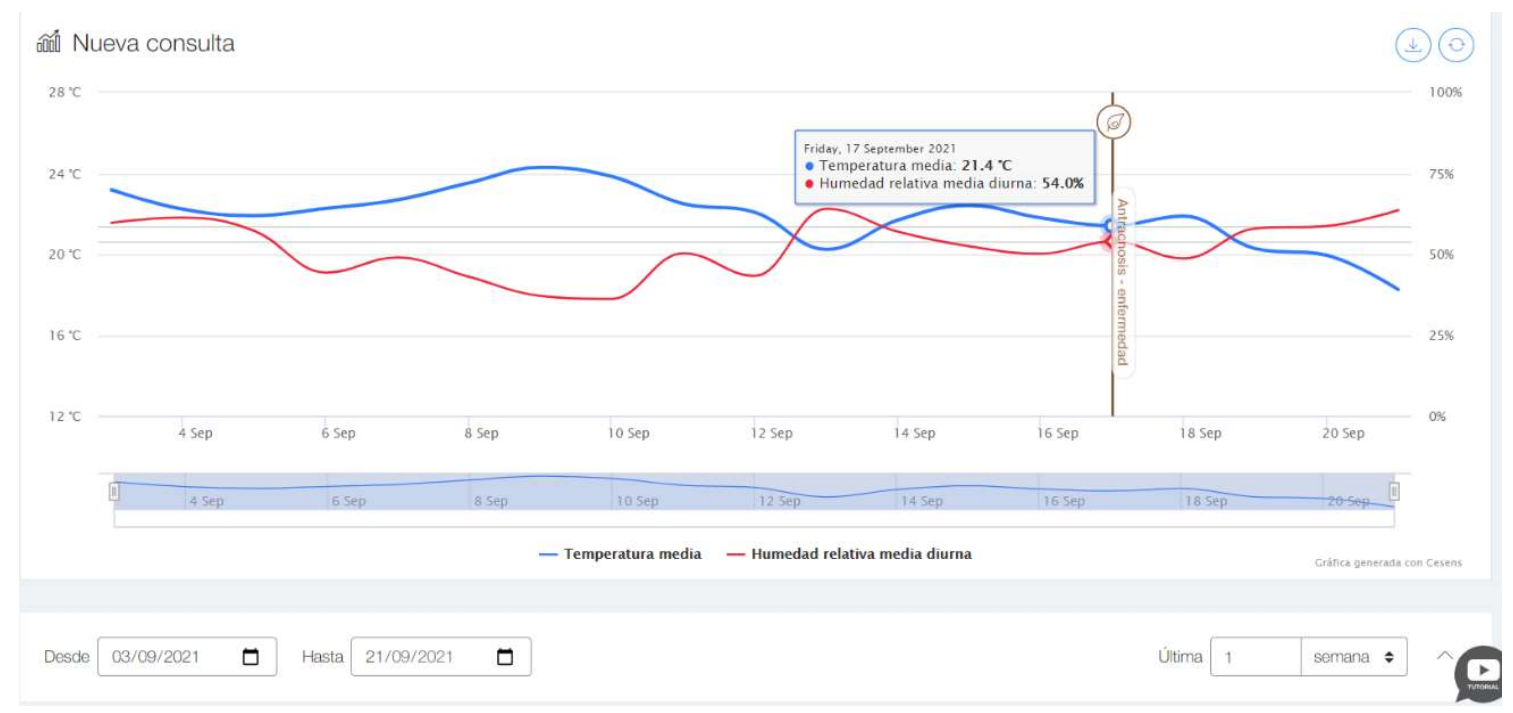

Figura 6. Evolución de temperatura y humedad y probabilidad de aparción de Antacnosis.

También, el cálculo de la integral térmica nos puede ayudar a calcular los ritmos vitales de plagas y enfermedades. Insectos, hongos y bacterias también responden y se desarrollan en función de una integral térmica. De esta forma, según las previsiones meteorológicas podemos 


\section{Congreso Nacional de Riegos CARTAGENA 2021}

anticiparnos a la aparición de una plaga o enfermedad y hacer el tratamiento oportuno de forma preventiva únicamente si se dan las condiciones idóneas.

\subsection{Control integral bajo una única plataforma;}

La plataforma web permite controlar todos los parámetros propios del cabezal, pero también posibilita poder conectar otros dispositivos bajo un mismo usuario.

Podemos disponer de datalogger repartidos por la explotación, monitorizando diferentes parámetros a lo largo de la misma y tenerlos vincularlos dentro de la misma plataforma web de control del riego y la fertirrigación para poder consultarlos de forma conjunta sin necesidad de disponer de diferentes plataformas.

Esto permite facilitar la experiencia de usuario en el manejo y consulta de los datos, no perder tiempo innecesario pasando de una plataforma a otra, y sobre todo, poder disponer y trabajar con los datos de varios dispositivos de forma autónoma y sencilla, tal y como se aprecia en la Figura 7.
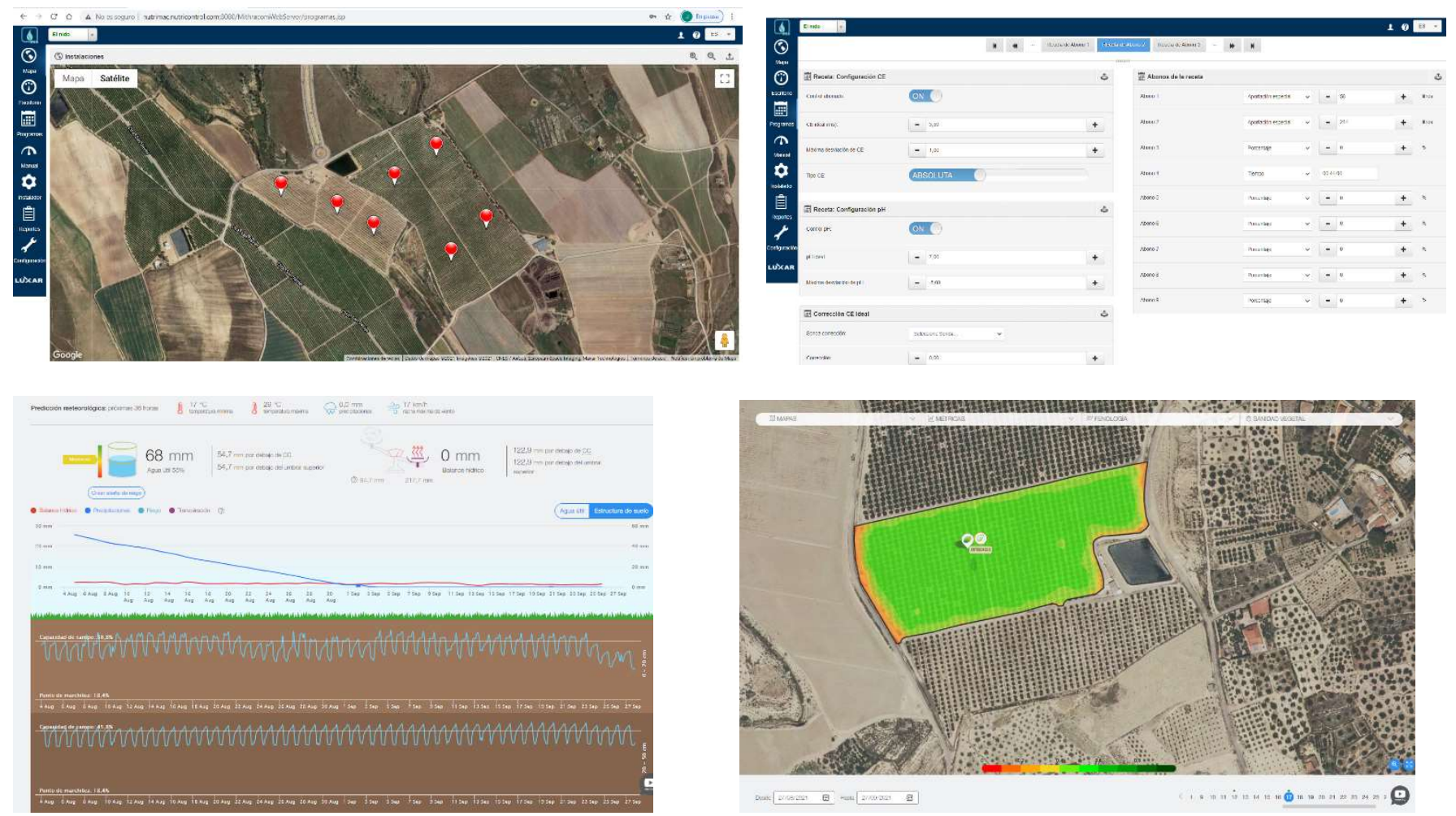

Figura 7. Integración de todas las medidas y control en plataforma web. 


\section{Conclusiones}

Nos encontramos en un cambio hacia la digitalización del campo y hacia una democratización de los datos por lo que, tenemos que aportar soluciones de valor y precisas que nos permitan elegir las estrategias más adecuadas para el manejo del riego y la fertirrigación.

Además, tenemos que apoyarnos en la aplicación de la tecnología y en el desarrollo de nuevos productos y sistemas encaminados a evolucionar y movernos para ser el cambio que queremos ver en la agricultura. Adaptándonos a las nuevas necesidades de la sociedad sin perder competitividad y que el agricultor pueda seguir siendo agricultor. Todo esto es imposible de alcanzar sin la utilización y desarrollo de tecnología en todos los procesos de producción de una explotación agrícola, la incorporación de sensórica y un trabajo posterior de analisis y evaluación de los datos generados con los sistemas instalados.

Para facilitar este trabajo, reducir costes energéticos, de fertilizantes y agua, se ha integrado toda la sensórica en un cabezal compacto que ha permitido ahorros energéticos de hasta un 35\%, un $20 \%$ en consumos hídricos y un $25 \%$ en ahorro de fertilizantes, constatado en diferentes cultivos. Asimismo, se ha facilitado al agricultor una plataforma amigable de fácil consulta que integra toda la información de los sensores, modelos predictivos y volcado sobre planos, para facilitar la toma de decisiones y cumplir con ello los objetivos de sostenibilidad marcados. 